
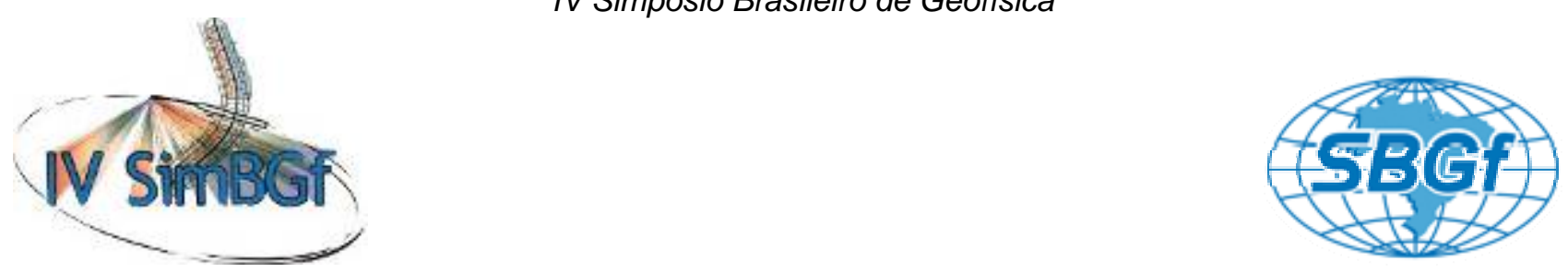

\title{
Análise e interpretação de lineamentos da região setentrional da Província Borborema, a partir de imagens Landsat e dados sismológicos
}

Adriana Chatack Carmelo*, Eduardo Sobral de Souza, Isabela Moreno Cordeiro de Souza, Elias Martins Guerra Prado, José Eduardo Pereira Soares, José Oswaldo de Araújo Filho

Instituto de Geociências/ Universidade de Brasília (chatack@unb.br)

Copyright 2010, SBGf - Sociedade Brasileira de Geofísica

Este texto foi preparado para a apresentação no IV Simpósio Brasileiro de Geofísica, Brasília, 14 a 17 de novembro de 2010. Seu conteúdo foi revisado pelo Comitê Técnico do IV SimBGf, mas não necessariamente representa a opinião da SBGf ou de seus associados. É proibida a reprodução total ou parcial deste material para propósitos comerciais sem prévia autorização da SBGf.

\section{Resumo}

A Província Borborema apresenta diferentes domínios estruturais que resultaram da ação de processos de sua formação a partir do Neoproterozóico. Com intuito de contribuir com informações sobre a Província, este estudo mostra a integração de lineamentos extraídos de imagens de satélite e dados de anisotropia crustal gerados a partir de levantamento sísmico. Os resultados foram sumarizados a partir de diagramas de roseta das principais direções dos lineamentos e dos resultados de anisotropia obtidos para as estações sismográficas.

\section{Introdução}

Este trabalho é uma contribuição à caracterização estrutural dos domínios Médio Coreaú, Ceará Central, Orós-Jaguaribe e Rio Grande do Norte, Província Borborema, região setentrional do estado do Ceará. A caracterização da área ocorreu a partir da análise de lineamentos extraídos de imagens Landsat/TM, na escala 1:200.000, e de direção de anisotropia crustal, obtida a partir de levantamento de refração sísmica profunda (INCT de Estudos Tectônicos/CNPq).

A determinação das direções principais de falhamentos e fraturas que caracterizam o domínio rúptil de uma região pode auxiliar na definição dos domínios tectônicos regionais, bem como contribuir para o entendimento da dinâmica dos aqüíferos fraturados e para a definição das direções principais de fraqueza, que potencialmente se relacionam com a sismidade recente.

\section{Área de Estudo}

A Província Borborema, Nordeste do Brasil, abrange os estados do Piauí, Ceará, Rio Grande do Norte, Paraíba, Pernambuco, Alagoas e Sergipe. Foi formada no Neoproterozóico, como parte do processo de formação da porção ocidental do supercontinente Gondwana, resultado da convergência e aglutinação dos crátons
Oeste-Africano, Amazônico e São Francisco-Congo (Castaing et al. 1994; Trompete 1994). A Província é caracterizada pela aglutinação de distintos domínios amalgamados ao longo de extensas zonas de cisalhamento de direções preferenciais NE-SW e E-W.

A área de estudo ocupa a porção setentrional da Província Borborema, abrangendo de NW para SE os domínios estruturais Médio Coreaú (DMC), Ceará Central, subdomínios Arco Santa Quitéria (ASQ) e SE Ceará Central (SE_CC), Orós-Jaguaribe (DOJ) e subdomínio NW Rio Grande do Norte (DRGN). Estes terrenos são delimitados pelas zonas de cisalhamento Sobral-Pedro II, Senador Pompeu e Portalegre, respectivamente.

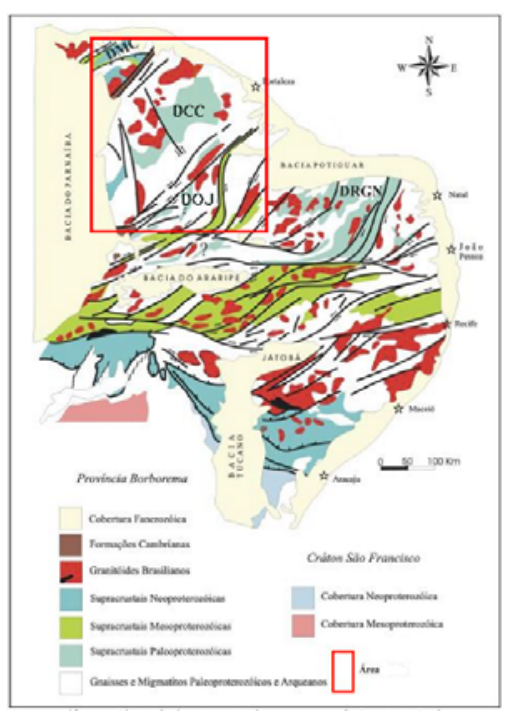

Figura 1 - Mapa de localização da área de estudo no contexto dos domínios tectônico. (Fonte: Santos et al. 2004).

\section{Metodologia}

As imagens Landsat/ TM foram adquiridas no sítio www.dsr.inpe.br, nas 6 bandas espectrais que constituem o espectro de radiação eletromagnética refletida, perfazendo um mosaico de 9 cenas com cerca de $180.000 \mathrm{~km}^{2}$ (Figura 2). 


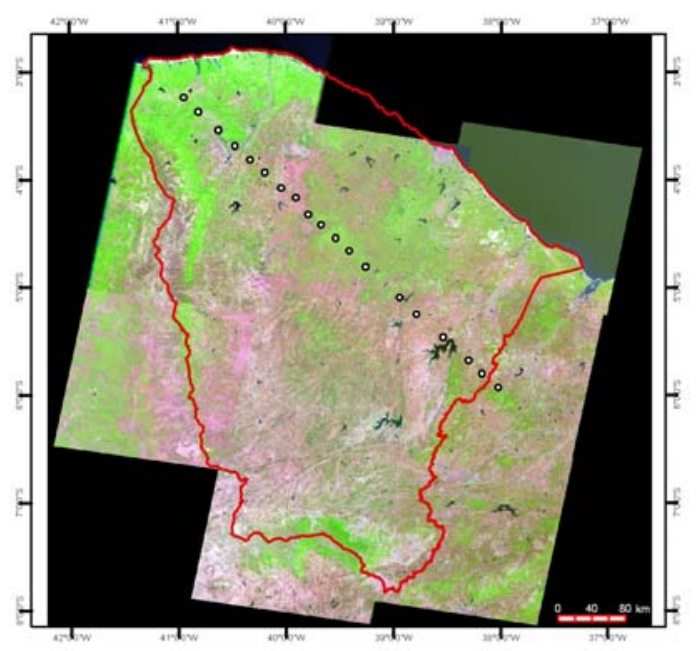

Figura 2 - Imagem Landsat/TM (RGB/ 543) do Estado do Ceará com a localização das estações sismográficas de três componentes (19 estações) utilizadas para determinação das direções de anisotropia crustal.

Foram extraídos os lineamentos a partir de composições coloridas RGB (6 bandas espectrais), com base nos seguintes critérios (Carmelo 2002):

i) feição linear que apresenta arranjo retilíneo ou suavemente curvo;

ii) quebra de relevo positivo;

iii) lineamentos curvos definidos por conjunto de retas descontínuas; e

iv) classificação dos lineamentos em lineamentos pequenos $(1 \mathrm{~km} \leq \mathrm{lp} \leq 4 \mathrm{~km})$, lineamentos médios $(4 \mathrm{~km}$ $<\mathrm{Im} \leq 16 \mathrm{~km})$ e lineamentos grandes $(\mathrm{lg}>16 \mathrm{~km})$.

A população total foi de aproximadamente 3.000 lineamentos analisados a partir de diagramas de roseta gerados para os domínios Médio Coreaú (DMC), Ceará Central, subdomínio Arco Santa Quitéria (ASQ) e subdomínio SE Ceará Central (SE_CC), Orós-Jaguaribe
(DOJ) e subdomínio NW Rio Grande do Norte (DRGN) (Figura 2).

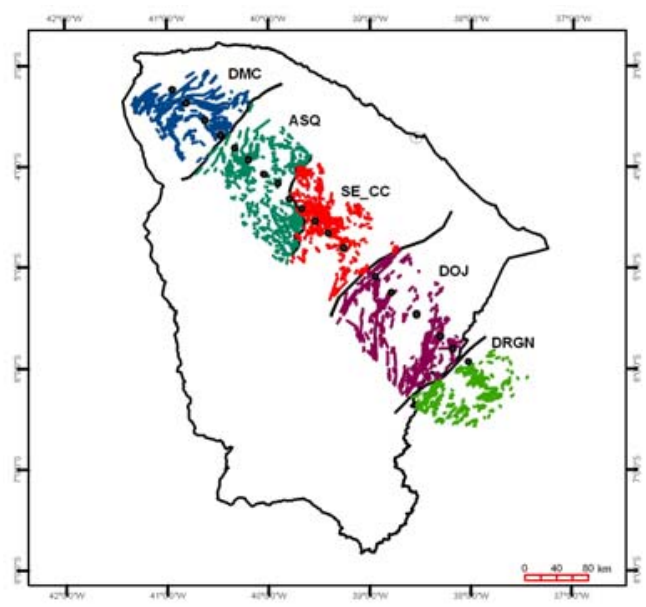

Figura 3 - Mapa com os lineamentos extraídos das imagens Landsat/ TM e apresentados por domínios.

A direção de anisotropia da crosta foi obtida a partir da determinação da (re-)polarização das ondas $S$, utilizando registros de explosões, de levantamento de refração sísmica profunda, em 19 estações sismográficas de período curto (3 componentes) distribuídas ao longo de um perfil de aproximadamente $400 \mathrm{~km}$ na direção NW-SE (Figura 2).

Os componentes horizontais de registro foram rotacionados até se obter a direção de maior energia da onda S (maior amplitude), caracterizando a direção de anisotropia do meio.

Para cada domínio, foi gerado: i) diagrama de roseta que sumariza as direções principais dos lineamentos, mostrados na cor verde, e ii) diagrama de roseta que sumariza os resultados de anisotropia obtidos para as estações sismográficas, mostrados na cor azul (Figura 3). 


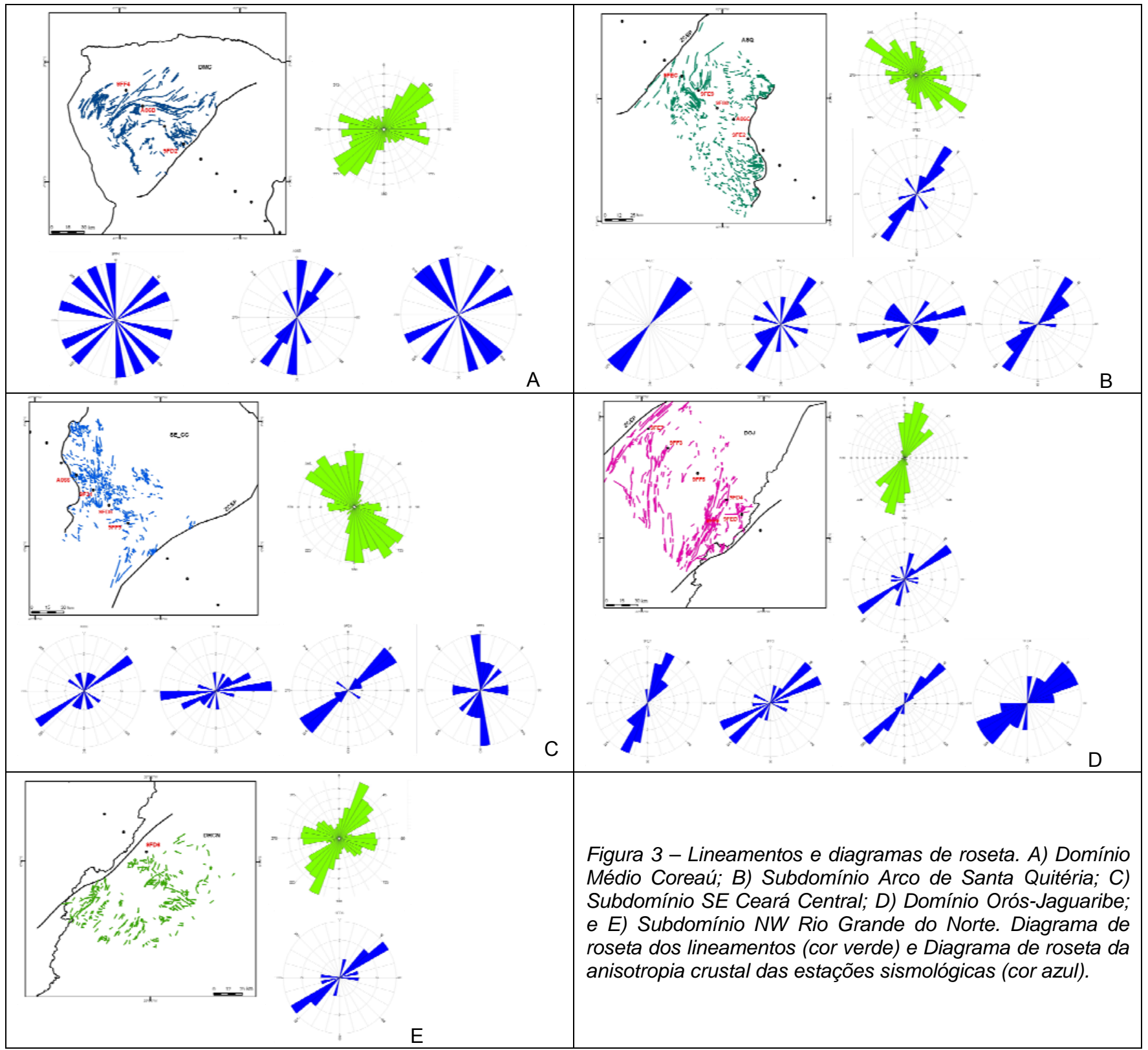

\section{Resultados}

A Tabela 1 sumariza os resultados obtidos.

No domínio Médio Coreaú os lineamentos apresentam direção preferencial NE-SW e, subordinadamente, direção aproximada E-W. Os resultados de anisotropia mostram dispersão grande, entretanto, as direções NESW e N-S são as principais.

O subdomínio Arco Santa Quitéria é caracterizado por lineamentos com direção NW-SW e, de forma subordinada, lineamentos N-S e E-W. A anisotropia apresenta direção NE-SW e secundariamente direção ENE-WSW.
No subdomínio SE Ceará Central os lineamentos se concentram ao longo de uma faixa definida entre as direções N-S e NW-SE e, subordinadamente, na direção $\mathrm{E}-\mathrm{W}$. Os resultados de anisotropia crustal têm direções NE-SW e N-S e subordinadamente direção E-W.

O domínio Orós-Jaguaribe apresenta lineamentos concentrados na direção NNE-SSW. Para os dados de anisotropia, a direção principal é NE-SW, secundariamente NNE-SSW.

O subdomínio NW Rio Grande do Norte mostra os lineamentos com direção principal NE-SW e subordinadamente E-W. A anisotropia crustal apresenta direção NE-SW e, secundariamente, em torno de E-W. 
Tabela 1 - Direções dos lineamentos e de anisotropia crustal, por domínios estruturais, em ordem decrescente de incidência.

\begin{tabular}{|c|c|c|c|}
\hline Domínios & Lineamentos & Anisotropia & Comum \\
\hline DMC & $\begin{array}{c}\text { NE-SW } \\
\text { ENE-WSW } \\
\text { ESE-WNW }\end{array}$ & $\begin{array}{c}\text { NE-SW } \\
\text { N-S } \\
\text { NW-SE }\end{array}$ & NE-SW \\
\hline ASQ & $\begin{array}{c}\text { NW-SE } \\
\text { N-S } \\
\text { E-W }\end{array}$ & $\begin{array}{c}\text { NE-SW } \\
\text { ENE-WSW }\end{array}$ & ENE-WSW \\
\hline SE_CC & $\begin{array}{c}\mathrm{N}-\mathrm{S} \\
\mathrm{NW}-\mathrm{SE} \\
\mathrm{E}-\mathrm{W}\end{array}$ & $\begin{array}{c}\text { NE-SW } \\
\text { N-S } \\
\text { E-W }\end{array}$ & $\begin{array}{l}\mathrm{N}-\mathrm{S} \\
\mathrm{E}-\mathrm{W}\end{array}$ \\
\hline DOJ & NNE-SSW & $\begin{array}{c}\text { NE-SW } \\
\text { NNE-SSW }\end{array}$ & NNE-SSW \\
\hline DRGN & $\begin{array}{c}\text { NE-SW } \\
\text { E-W }\end{array}$ & $\begin{array}{c}\text { NE-SW } \\
\text { E-W }\end{array}$ & $\begin{array}{c}\text { NE-SW } \\
\text { E-W }\end{array}$ \\
\hline
\end{tabular}

\section{Discussão e Conclusão}

Os lineamentos de superfície apresentam tendência de sofrerem variações na direção preferencial de alinhamento de acordo com os domínios estruturais de onde foram extraídos, mostrando que os diferentes domínios apresentam características estruturais distintas.

A anisotropia crustal apresenta direção preferencial NESW, independente do domínio estrutural analisado, mostrando que a re-polarização das ondas $S$ não é influenciada, prioritariamente, pelas direções impressas em superfície.

A direção NE-SW, bem marcada pela anisotropia, coincide com a direção das falhas de rifteamento da Bacia Potiguar, geradas pelo estiramento litosférico imposto à Província Borborema durante o Cretáceo (Soares e Fuck 2010). Grosso modo, nas regiões de maior estiramento, representadas pelo domínio OrósJaguaribe e subdomínio SW Rio Grande do Norte, as direções dos lineamentos e de anisotropia tendem a convergir.

De forma geral, os resultados obtidos mostram que os lineamentos representam, prioritariamente, as deformações impressas nos terrenos ao longo de sua evolução geológica, refletindo a herança de deformações tectônicas. Entretanto, as direções de anisotropia traduzem a direção de planos de fraqueza impressos, provavelmente, pelo último grande evento tectônico a afetar a região.

\section{Agradecimentos}

Os autores agradecem ao Projeto Estudos Geofísicos e Tectônicos na Província Borborema (Instituto do Milênio CNPq/MCT) pela disponibilização dos dados sísmicos de refração profunda.

\section{Referências}

Almeida, F. F. M.; Hasui, Y.; Brito Neves, B. B.; Fuck, R. A. 1981. Brazilian structural provinces: $\mathrm{Na}$ introduction. Earth-Science Reviews, 17:1-29.

Carmelo, A. C. 2002. Caracterização de Aquíferos Fraturados por Integração de Informações Geológicas e Geofísicas. Tese de Doutorado. IG/ UnB. Brasília. DF. $153 p$.

Castaing, C. F.; Thiélblemont, T. C.; Chevremont, P. 1994. Paleographical reconstruction of the Pan-African/ Brasiliano orogen: closure of an oceanic domain or intracontinental convergence between major blocks. Precambrian Research, 69:327-344.

Trompete, R. 1994. Geology of western Gondwana, PanAfrican/ Brasiliano Aggregation of South America and Africa. A. A. Balkema, Rotterdam, Brookfield, 350p.

Santos, T. J. S.; Fetter, A.; Hackspacher, P.; Van Schums, W.; Nogueira Neto, J. 2004. Structural and Geochronological studies of Médio Coreaú Domain, NE Brazil: Constraints on Brasiliano/ Pan-Africano tectonic evolution in the NW part of the Borborema Province. In: Amaral, W. S. 2007. Integração de dados aerogeofísicos e geológicos e sua contribuição a evolução geodinâmica do Domínio Ceará Central, Província Borborema. Dissertação de Mestrado. IG/ UNICAMP. Campinas. SP. $113 p$.

Soares, J.E.P. e Fuck, R.A., 2010. Lithosphere of the Borborema Province, NE Brazil: insights from geophysical data, Meeting of the Americas, Foz do Iguaçu, PR, Brasil, T12A-05. 